\title{
The Robotic Palomar 60-Inch Optical Afterglow Catalog: 2007
}

\author{
Janos Kelemen*, Brad Cenko ${ }^{\dagger}$ and Derek Fox ** \\ ${ }^{*}$ Konkoly Observatory \\ ${ }^{\dagger}$ Caltech SLR \\ ${ }^{* *}$ Pennsylvania State University
}

\begin{abstract}
We present here the photometric results of the Robotic Palomar 60-inch Telescope (P60) observations for GRB optical afterglows. The quick response time and the size of the telescope allows us to observe the optical transients through V, R, I, g', i', z' filters, sometimes as early as 0.01 day after the trigger. Comparing the data obtained with the various filters we were able to compute color indices for the 1 day after the trigger epoch. The observations lasts until the OT fades below the detection threshold.
\end{abstract}

Keywords: GRB, optical afterglow, photometry

PACS: $98.70 . \mathrm{Rz}, 95.85 . \mathrm{Kr}, 95.85 . \mathrm{Pw}$

\section{INTRODUCTION}

In the present work we summarize the GRB OT observations of the 2005-2007 period respecting the color behaviours of the optical transients.

In this paper we focus on the colors of the observed OT-s. During the majority of the P60 observations the data were obtained through g', r', i', z' filters. Although the datasets are limited i.e. there are no long photometric series we could derive the decay rate of the OT in the specific color bands and therefore we were able to compute the extrapolated brightnesses for the "1 day after the trigger" epoch. From the computed colors for this epoch we derived color indices in order to compare the color behaviour of the various events. In the cases when redshift measurements were available for the GRB sources we constructed the color-redshift diagrams as well.

\section{THE DATA}

All the data presented here were obtained with the aid of the Robotic 60-inch Telescope at Palomar Observatory (P60) [1]. For the photometric data reduction the aperture photometry (ATV software package (IDL)) method was used. The standard stars were selected from the USNO or from the SDSS catalogue. From the USNO magnitudes the g', r', i', z' magnitudes were computed according to Jordi et al. 2006 [2] with the modifications made by Derek Fox.

In the cases when both USNO and SDSS comparison stars were available for the photometry of the GRB OT-s we compared the USNO based g', r', i', z' magnitudes to the SDSS values respectively. The overall accuracy of our GRB OT photometry is in order of tenths of magnitude due to the low $\mathrm{S} / \mathrm{N}$ ratio near to the detection limit of the $\mathrm{CCD}$ camera.

\section{THE RESULTS}

For the well observed GRB OT-s we determined the coefficients of the powerlaw decay in the various color bands. Table 1. presents the list of the computed powerlaw decay index $(\alpha)$ and the extrapolated magnitudes in the individual color bands for the 1 day after trigger epoch. Table 2. shows the "1 day after the trigger" epoch g'-r', r'-i', i'-z' color indices and the available redshift values. Based on the previous P60 observations data we have to mention that in our 
sample according to the optical to X-ray spectral index $\beta(\mathrm{OX})$ significant fraction of the events belongs to the "dark bursts" Cenko et al. $2008[3]$ category $(\beta(\mathrm{OX})<0.5)$.

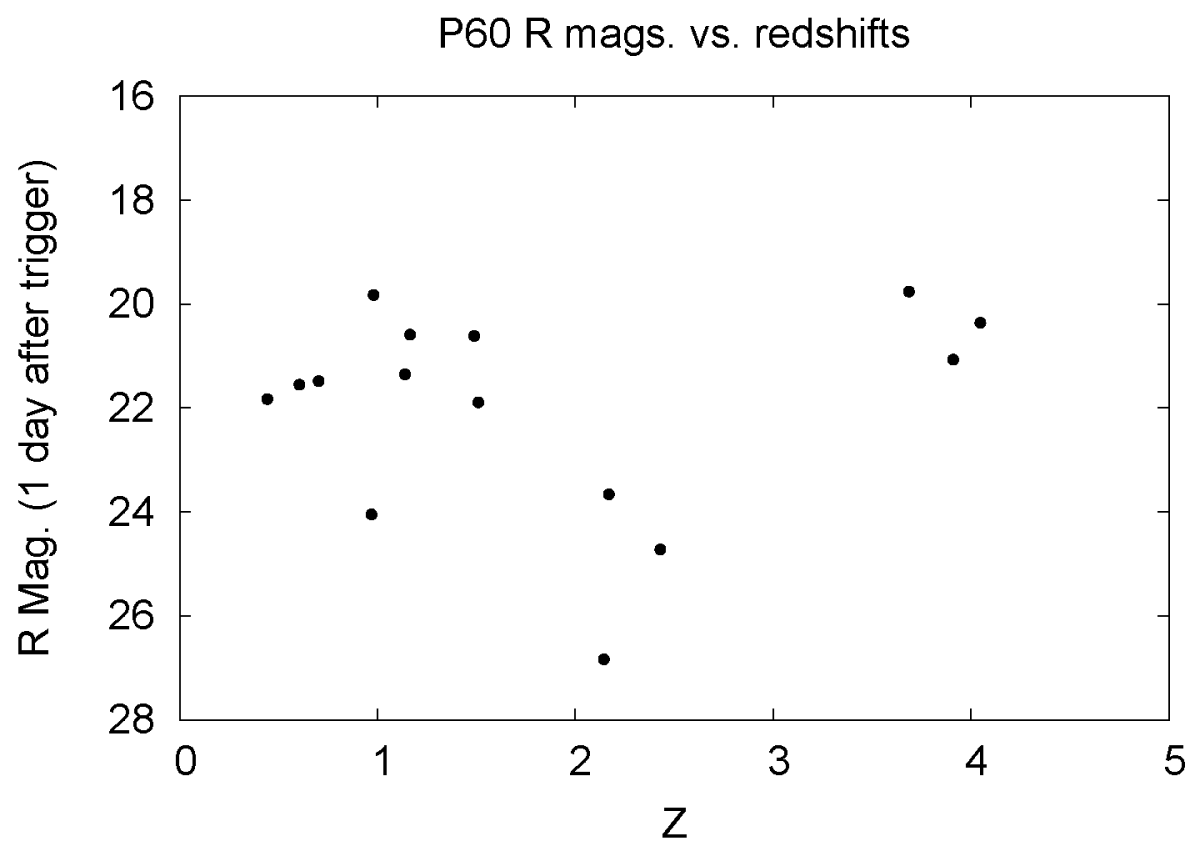

FIGURE 1. R mag. - Z plot. There is a clearly visible difference between the $\mathrm{R}$ brightness distribution of the lower redshift (left group) and higher redshift GRB-s (right group).

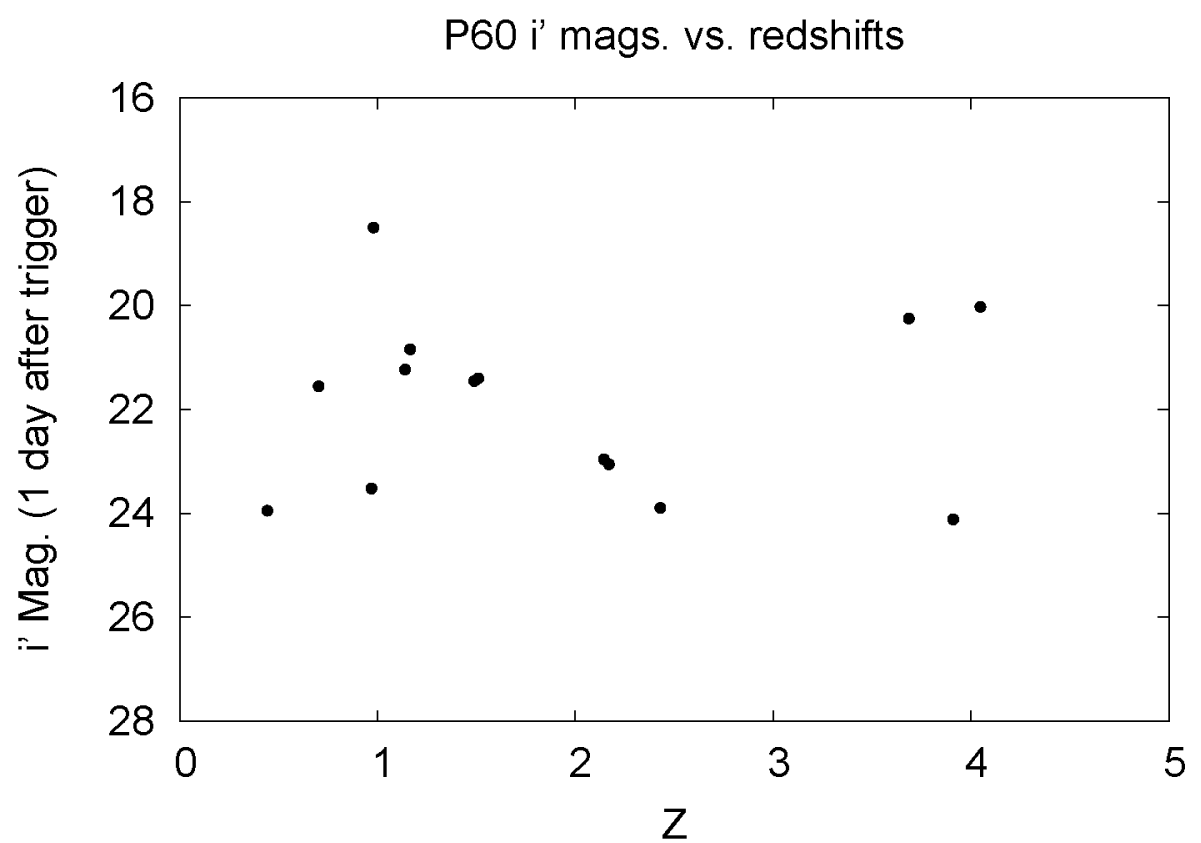

FIGURE 2. i' mag. - Z plot. The difference between the lower redshift (left group) and higher redshift GRB-s (right group) is remarkable. 


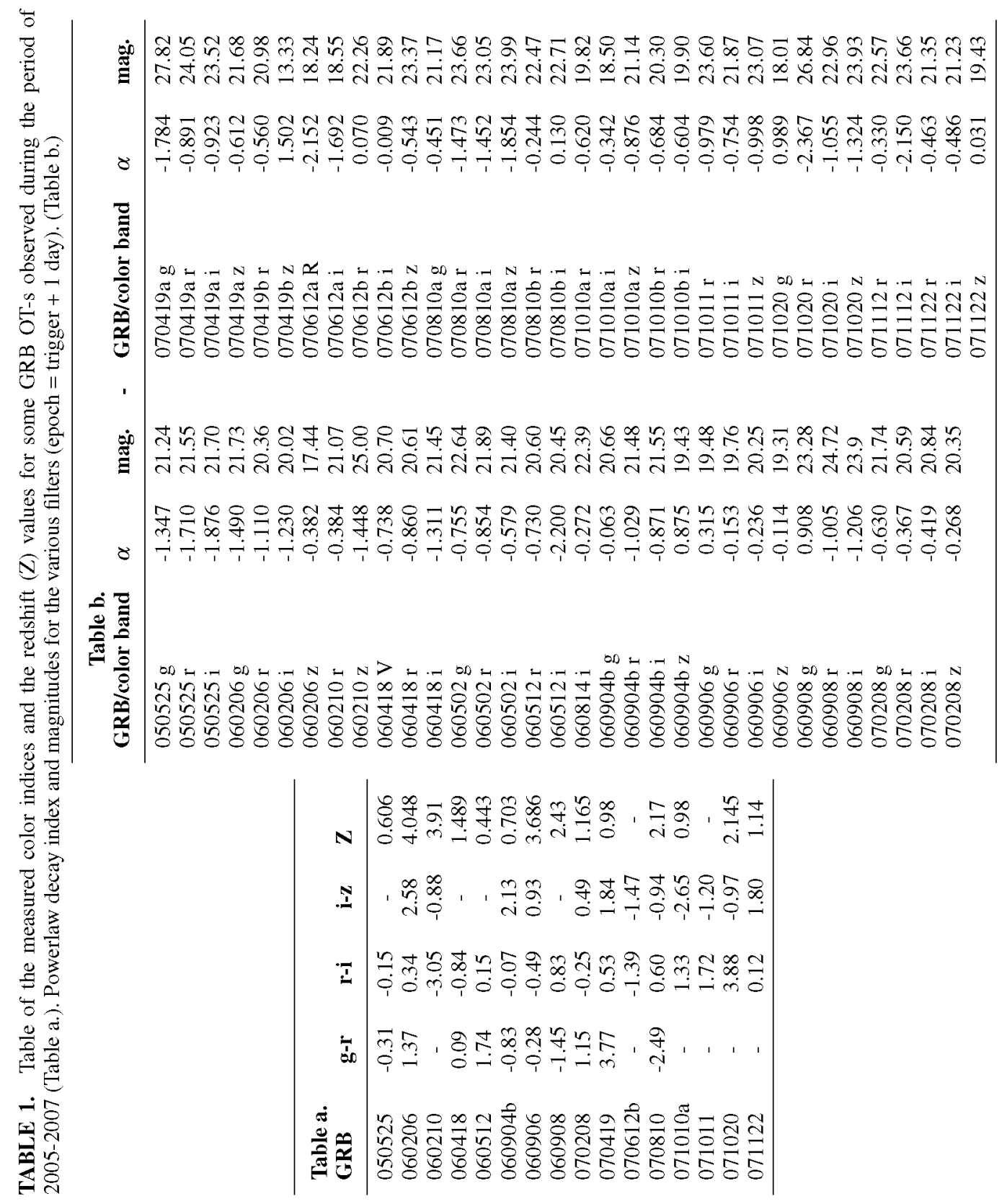




\section{CONCLUSIONS}

Based on the good results from our P60 photometry we plan to continue our GRB OT observations. According to our recent results the issue of the GRB colors and the investigation of the color - redshift relations needs much more efforts.

The results for the year 2008 are in preparation. We plan further investigation and refinement of the transformation coefficients between the USNO and SDSS catalogue and the P60 photometric system as well.

\section{ACKNOWLEDGMENTS}

This research was partially supported by the Hungarian Space Office (Janos Kelemen)

\section{REFERENCES}

1. S. B. Cenko, et al., PASP Vol.118, 2006, pp. 1396-1406.

2. K. Jordi, and E. K. Grebel, and K. Ammon, Astronomy and Astrophysics Vol.460, 2006, pp. 339-347.

3. S. B. Cenko, et al., submitted to ApJ, 2008, pp. 889-900. 